\title{
Case Title
}

Scoping Review: Online gaming interventions for congenital heart patients

Author Name(s)

Suzanne Fredericks

Author Affiliation \& Country of Affiliation

Ryerson University

Canada

Lead Author Email Address

Email: sfrederi@ryerson.ca

Discipline: D4 [please do not alter]

\section{Sub-discipline}

Nursing [SD-Hlth-12]

\section{Academic Level}

Introductory Undergraduate

\section{Contributor Biographies}

Dr. Suzanne Fredericks is a Professor and the Graduate Program Director in the Daphne Cockwell School of Nursing at Ryerson University. Dr. Fredericks is also an Affiliate Scientist (May 2016-present) at the University Health Network's Toronto General Research Institute (http://www.uhnresearch.ca/researcher/suzanne-fredericks-0).

\section{Published Articles}

[Fredericks, S., Lapum, L., Policelli, J. (Accepted). Applying the Reach, Efficacy, Adoption, Implementation, and Maintenance framework to the evaluation 


\begin{abstract}
Online patient education gaming interventions should be designed for the adult cohort with congenital heart disease since current resources are not optimal. To date, these types of interventions are currently being used throughout the healthcare environment. Even though my area of expertise encompasses the design and evaluation of patient education interventions, I have primarily focused on face-to-face and/or telephone based interventions. My knowledge in the area of online gaming is severely limited. As a result a scoping review was undertaken in order for the acquisition of a better understanding of what online gaming is; its associated characteristics, and the most common outcomes associated with these interventions. This case study presented an overview of a scoping review that was conducted with the main goal of summarizing and disseminating research findings, identifying research gaps, and making recommendations for future intervention design and evaluation. The stages for the conducting a scoping review were presented along with some practical lessons learned.
\end{abstract}

\title{
Learning Outcomes
}

By the end of this case students should be able to:

- Understand when and how to conduct scoping reviews

- Recognize the limitations and benefits associated with conducting a scoping review

- Integrate and apply findings from scoping reviews into practice

\section{Case Study}


Congenital heart disease (CHD) is a condition present at birth that encompasses a number of diseases involving the heart (Verheugt et al., 2010). Over the years, the diagnosis and treatment of CHD has significantly advanced, allowing individuals to live into adulthood (Heery, Sheehan, While, Coyne, 2015). As a result, lifelong complications exist which results in individuals requiring additional care throughout their lifespan. However, their ability engage in employment is significantly limited, as many of these individuals tend to be too sick to work, and thus are more likely to be on permanent disability. As well, the reoccurrence and/or exacerbation of symptoms results in increased use of the health resources, which often times is significantly higher than that of the average individual (Silversides et al., 2010). As a result, the combination of the onset and/or aggravation of symptoms and limited work connections has led to decrease social interactions among these individuals.

Resources to support persons with CHD are limited and consist primarily of patient education booklets (Triedman \& Newburger, 2016). However, these resources fail to provide opportunities for social and/or group interaction. Online gaming is an alternative resource for patient education interventions. This intervention provides a platform for knowledge acquisition, retention, and recall and can also be designed to create a forum for social interaction and engagement, as well as entertainment (Taekman \& Shelley, 2010; Freddolino $\&$ Blaschke, 2008). To date, online gaming interventions have been used throughout the healthcare environment, however no extensive review has been conducted to determine the characteristics of these interventions, such as specific features, length of time for delivery, process related to how it is delivered, who can access it, type of content delivered, and how often gaming intervention can be accessed, in addition to its effectiveness. This information is needed to effectively design, implement, and begin to evaluate the effectiveness of online gaming interventions delivered to individuals diagnosed and living with CHD. 
The purpose of this scoping review is to determine the characteristics of online gaming interventions for patients in the context of healthcare.

\section{Research Practicalities}

My expertise is in the area of designing and evaluating face-to-face and/or telephone based patient education interventions. I have been working with these interventions for the past 7 years. Additionally, I have advanced clinical trials intervention research training with a focus on patient education and have published over 50 manuscripts in this area. I mention this because with all of my experience and knowledge in the area of patient education interventions delivered via face-to-face and/or by telephone, I have come to realize that I basically have little to no knowledge of other forms of patient education interventions delivered using alternative mediums such as through the use of an online gaming platform. This became most evident during a recent encounter that I had with individuals from the Canadian Congenital Heart $(\mathrm{CCH})$ group. Representatives from this group approached a faculty colleague and myself and requested that we assist them in the leading of a number of studies that address online gaming interventions for the $\mathrm{CCH}$ adult. The $\mathrm{CCH}$ representatives stated that currently minimal resources were available for adults diagnosed and living with congenital heart disease. They were reaching out to us, to determine if we could help them to design, implement, and evaluate an online gaming intervention that would be meaningful and relevant to their cohort. The impetus for the request was the feelings of helplessness experienced by these individuals due to the lack of resources that are made available to them.

The discussion had peaked our interest. Since we did not have a good understanding of online gaming patient education based interventions; we were unsure as to where to begin in terms of the type of intervention that we should be designing. As a result we decided to engage in a scoping review that would allow us to have a better understanding of what online 
gaming is; its associated characteristics, and the most common outcomes associated with these interventions.

\section{Research Design}

Scoping reviews are typically used to gain a better understanding of a concept, in this case, online gaming patient education interventions, and to evaluate where research on a topic has or has not been completed. It consists of a quick gathering of the literature and provides a summary of previous research activity, while identifying gaps in the research (Arksey \& O’Malley, 2005). In general, scoping reviews serve to map the key concepts that underpin a research area, along with the main sources and types of evidence in areas that have not been examined extensively (Arksey \& O'Malley). It is important to note that even though scoping reviews allow for the identification of gaps in the literature, it does not identify research gaps where the research is of poor quality, as quality assessment are not a component of the scoping review process (Arksey \& O’Malley, 2005; p. 22). Arksey \& O’Malley (2005) reasoned that scoping reviews are a preliminary methodology in the hierarch of reviews (scoping review, evidence assessment, and systematic review), with systematic reviews being the most rigorous form of a review (Arksey \& O’Malley).

The process for conducting a scoping review, as outlined by Arksey and O’Malley' (2005) consists of five stages that include: identifying the research question to be addressed; identifying works that are of relevance to the research question; screening and selecting works to be included in the review; charting the information and data within the included studies; and collating, summarizing, and reporting results of the review.

\section{"Method" in Action}

According to Arksey and O’Malley, all aspects of the research area should be considered before formulating the research question, to ensure the research question is sufficiently broad. Taking into account the expertise of our research team, and after 
conducting an initial scan of the literature, we generated the following research question: What are the characteristics of online gaming interventions for patients in the context of healthcare? This broad question allowed us to include diverse literature, with the goal of informing and developing new directions for nursing research.

The second stage consisted of identifying works that are of relevance to the research question. We began by consulting with librarians and online gaming experts. Their involvement allowed us to collect feedback about the research question and sources of literature. The search was limited to articles written in English, as this study was being conducted in English. Additionally, articles published between 2001 and 2016 were included. This timeframe was selected, as there has been a significant increase in the development, application, and evaluation of online gaming interventions in health care settings during this period. This classification of study participant was intentionally kept broad to ensure the largest segment of the literature was reviewed. All studies included in this review had to discuss and/or evaluate an online gaming intervention. Furthermore, studies that used either qualitative or quantitative designs were also included in this review. As well, all outcomes were included, to determine the type of outcome that is typically evaluated with online gaming interventions.

The third stage consisted of screening and selecting the appropriate literature. This was achieved through the independent review of all articles by the various members of the research team that consisted of a principal investigator, a co-investigator, and two research assistants. The screening process consisted of the research assistants reviewing the title, abstract, and then the full text. In case of any uncertainty about a paper based on its title and the abstract, the first author reviewed the full text. No discrepancies appeared at the final stage of screening. 
The fourth stage associated with the conduct of a scoping review is that of charting. This stage consists of mapping the information obtained. In other words, taking the large volume of information and presenting diagrammatically, how the decisions that were made to yield the final sample. In this scoping review, the search initially yielded 26, 500 articles. In order to refine the search, only articles that were available via electronic medium were considered. This allowed for a more in-depth analysis of the articles that examined or described online gaming in healthcare, in a timely manner. Thus, from the 26, 500 titles, 73 abstracts were assessed, with the final screening process yielding a total of 42 papers that were included. This process of elimination is presented diagrammatically in the attached Figure 1.

The final stage associated with conducting scoping reviews consisted of collating, summarizing, and reporting of the results. With regards to this systematic review, a multistage process to was used to collate and summarize the data. Common descriptive demographic information from individual articles based on specified categories: author(s), publication date (year), percentage of immigrant women included in the study, participant's countries of origin, location/country of the first author, literature descriptors (research/theory/commentary/summary of literature), type of study design (qualitative, quantitative, mixed method), setting for data collection, type of data collected, type of CVD intervention offered to immigrant women, and the study findings were extracted and placed into a table format. The data was then transferred to a data management statistical spreadsheet, in which statistical analyses were then computed. Results suggest, even though much of the online gaming interventions were designed with the intent that they be used with individuals experiencing chronic illnesses, many of these interventions were evaluated using healthy, young adults, and in many cases adolescents. As well, no study used interventions that incorporated multi-player online gaming options. Additional testing of these 
interventions using a variety of populations, in a number of settings, and incorporating multiplayer options is required.

\section{Practical Lessons Learned}

The use of a scoping review was appropriate for the purpose that we had established, in that it provided us with an overview of online patient education gaming interventions. Even though much of the literature classify scoping reviews as less rigorous and methodologically sound as compared to systematic reviews due to the lack of methodological critique and quality assessment; these types of studies still require a fair bit of work. In reading the literature pertaining to scoping reviews, there is a misconception that these types of studies are quick and easy to conduct. There is still a significant amount of organization and pre-planning that is needed prior to the conduct of scoping reviews. Establishing clear research questions, selection criteria, identifying key informants, having a well versed knowledge of how to engage in literature searches and the use of data bases, as well as how to create and effectively use data management and statistical software are all important considerations prior to engaging in a scoping review.

If you are able to be involved in the conduct of a scoping review, even if it is from the perspective of a research assistant, I would strongly encourage you to take advantage of this opportunity, as it will provide you with skills that you can transfer into your clinical and professional work environment.

\section{Conclusions}

Online patient education gaming interventions should be designed for the adult cohort with congenital heart disease since current resources are not optimal. To date, these types of interventions have been used throughout the healthcare environment, however no extensive review has been conducted to determine their characteristics. This case study presented a synthesis of a scoping review that was conducted with the main goal of summarizing and 
disseminating research findings, identifying research gaps, and making recommendations for future intervention design and evaluation. The stages for the conducting a scoping review were presented along with some practical lessons learned.

\section{Exercises and Discussion Questions}

1) What are scoping reviews?

2) How do scoping reviews differ from systematic reviews?

3) What are the main features associated with the conduct of a scoping review?

4) What is the most significant benefit associated with conducting scoping reviews?

5) What is the most significant limitation associated with conducting scoping reviews?

\section{Further Readings}

Arksey, H., \& O'Malley, L. (2005). Scoping studies: towards a methodological framework. International journal of social research methodology, 8(1), 19-32.

Kastner, M., Tricco, A. C., Soobiah, C., Lillie, E., Perrier, L., Horsley, T., ... \& Straus, S. E. (2012). What is the most appropriate knowledge synthesis method to conduct a review? Protocol for a scoping review. BMC Medical Research Methodology, 12(1), 1.

Levac, D., Colquhoun, H., \& O'Brien, K. K. (2010). Scoping studies: advancing the methodology. Implementation Science, 5(1), 1.

\section{Web Resources}

None

\section{References}


Arksey, H., \& O'Malley, L. (2005). Scoping studies: towards a methodological framework. International journal of social research methodology, 8(1), 19-32.

Freddolino, P. P., \& Blaschke, C. M. (2008). Therapeutic applications of online gaming. Journal of Technology in Human Services, 26(2-4), 423-446.

Heery, E., Sheehan, A. M., While, A. E., \& Coyne, I. (2015). Experiences and outcomes of transition from pediatric to adult health care services for young people with congenital heart disease: a systematic review. Congenital heart disease, 10(5), 413-427.

Silversides, C. K., Marelli, A., Beauchesne, L., Dore, A., Kiess, M., Salehian, O., ... \& Khairy, P. (2010). Canadian Cardiovascular Society 2009 Consensus Conference on the management of adults with congenital heart disease: executive summary. Canadian Journal of Cardiology, 26(3), 143-150.

Taekman, J. M., \& Shelley, K. (2010). Virtual environments in healthcare: Immersion, disruption, and flow. International anesthesiology clinics, 48(3), 101-121.

Triedman, J. K., \& Newburger, J. W. (2016). Trends in Congenital Heart Disease The Next Decade. Circulation, 133(25), 2716-2733.

Verheugt, C. L., Uiterwaal, C. S., van der Velde, E. T., Meijboom, F. J., Pieper, P. G., van Dijk, A. P., ... \& Mulder, B. J. (2010). Mortality in adult congenital heart disease. European heart journal, ehq032. 